\title{
Status Epilepticus and Delirium Associated with Ertapenem in a Very Elderly Patient with Chronic Kidney Disease and Silent Ischaemic Cerebrovascular Disease
}

\author{
Huimin $\operatorname{Lin}^{1} \cdot$ Samuel T. H. Chew ${ }^{1}$
}

Published online: 23 November 2015

(c) The Author(s) 2015. This article is published with open access at Springerlink.com

\begin{abstract}
An 85-year-old female with chronic kidney disease and newly acquired seizures on oral phenytoin received intravenous ertapenem $500 \mathrm{mg}$ once daily for a urinary tract infection and bacteraemia involving extendedspectrum beta-lactamase (ESBL)-producing Escherichia coli. After three ertapenem doses, she developed seizures which self-aborted. Corrected phenytoin level was subtherapeutic initially, but became therapeutic following a phenytoin dose increase. On Day 14 of ertapenem, the patient suffered another episode of seizure. Ertapenem was stopped. On Day 15, she developed status epilepticus lasting 2 days, requiring intravenous lorazepam, sodium valproate and phenytoin. The last episode of seizure occurred 3 days after discontinuation of ertapenem, with a dramatic recovery of her Glasgow Coma Scale and resolution of delirium. At the time of writing, she has remained seizure free for over 2 years. A retrospective audit of the emergency department notes revealed that she had a course of ertapenem in another institution prior, which also led to two seizure episodes-once 8 days after starting ertapenem, another 3 days after stopping ertapenem. Despite using a renal-adjusted ertapenem dose, each dip in her creatinine clearance level was associated with seizures, preceded by delirium each time. Naranjo assessment score was 6 , suggesting a probable relationship between the seizures and ertapenem.
\end{abstract}

Samuel T. H. Chew

Samuel_Chew@cgh.com.sg

1 Changi General Hospital, Singapore, Singapore

\section{Key Points}

There is a recognizable clinical pattern in the manifestation of ertapenem-induced delirium and seizures in the high-risk, very elderly patient.

Discontinuation of ertapenem therapy should be considered if seizures develop and alternative antibacterial therapy used instead if available.

High index of suspicion is required to detect and report these unseen and highly complex adverse drug reactions in clinical practice.

Although the incidence of ertapenem-induced seizures is reportedly low in the general population, more work and studies may be required to quantify the risks for specific high-risk subgroups.

\section{Introduction}

Carbapenems are an important class of broad-spectrum antibacterials for the treatment of complicated and serious bacterial infections caused by Gram-positive and Gramnegative organisms, especially extended-spectrum betalactamase (ESBL)-producing Enterobacteriaceae. Ertapenem, a carbapenem, is advantageous in that it is long-acting and allows for a convenient, once-daily dosing. This results from the addition of a meta-substituted benzoic acid group, granting it greater lipophilicity and a net negative charge at physiological $\mathrm{pH}$, hence greater plasma protein binding ability compared with other carbapenems [1]. Its $1-\beta$ methyl substituent confers stability against hydrolysis by 
renal dehydropeptidase-1 (DHP-1), thus it does not require concurrent administration with a DHP-1 inhibitor such as cilastatin [1].

However, carbapenems have been widely implicated in causing neurotoxicity, particularly seizures [2-4]. Most reports of seizures were associated with the use of imipenem-cilastatin at rates varying from 3 to $33 \%$ while occurrence of seizures with other carbapenems, including ertapenem, were individually reported to be $<1 \%$ [3].

We present here a case of repeated ertapenem use in two separate admissions under different primary medical admitting teams in a very elderly patient ( $>80$ years old) with chronic kidney disease, low serum albumin and incidental findings of silent small vessel cerebrovascular disease resulting in delirium and seizures. Discontinuation of ertapenem therapy was followed by complete and sustained resolution of both delirium and seizures.

\section{Case Report}

An 85-year-old female with a history of recurrent urinary tract infection (UTI) on a background of stage 5 chronic kidney disease presented with low-grade fever to the emergency department at Changi General Hospital (CGH) on 18 July 2013. She had just been discharged from a subacute step down unit at $\mathrm{CGH} 2$ days prior.

Before her index admission to CGH on 9 May 2013, the patient lived at home with her family, was independent in her activities of daily living, continent and independently ambulant with a walking stick. She was on atenolol $100 \mathrm{mg}$ OM (every morning) and nifedipine LA $60 \mathrm{mg}$ OM for hypertension, simvastatin $5 \mathrm{mg} \mathrm{ON}$ (every night) for hyperlipidaemia, allopurinol $100 \mathrm{mg}$ OM for gout, prochlorperazine maleate $5 \mathrm{mg}$ TDS (three times a day) for dizziness, aqueous cream for dry skin, hypromellose eye drops, vitamin B complex tablet and calcium and vitamin $\mathrm{D}$ tablet from the outpatient polyclinic. Her baseline estimated creatinine clearance $(\mathrm{CrCl})$ by Cockcroft-Gault was $12-14 \mathrm{~mL} / \mathrm{min}$. She was transferred to a community hospital for further rehabilitation on 30 May 2013 but was readmitted to $\mathrm{CGH}$ on 3 July 2013 for new onset seizures after receiving 8 days of intravenous (IV) ertapenem for a UTI. She did not have a prior history of seizures. In our retrospective audit of the notes, she was noted to have acute-on-chronic kidney injury during her first seizures at the community hospital, with an estimated $\mathrm{CrCl}$ of $10 \mathrm{~mL} /$ min. Despite the small absolute change from $12-14$ to 10 , this is a difference of $\mathrm{CrCl}$ of $14.3-28.6 \%$ in relative terms.

IV piperacillin/tazobactam was started empirically on 18 July 2013 on the patient's third admission to CGH for presumed recurrent UTI and possible hospital-acquired infection. She was haemodynamically stable and only had an intermittent low-grade fever $<38{ }^{\circ} \mathrm{C}$ in the first 2 days of her admission. She was afebrile from Day 3 onwards and her white cell count had returned back to normal values by Day 4.

However, the antibacterial therapy was subsequently switched to IV ertapenem $500 \mathrm{mg}$ every $24 \mathrm{~h}$ (q24h) on Day 3 in view of positive urine and blood cultures which grew ESBL-producing Escherichia coli (E. coli). She was also found at the point of admission to have acute-onchronic kidney injury secondary to dehydration, for which she was started on IV hydration.

Subsequent sensitivity testing revealed that the ESBLproducing $E$. coli was sensitive to

1. Amikacin.

2. Imipenem.

3. Ertapenem.

4. Meropenem.

5. Piperacillin/tazobactam.

Upon receiving three doses of ertapenem, the patient experienced an episode of self-aborted seizure with stiffness and jerking of all four limbs for a few seconds, followed by post-ictal drowsiness (Glasgow Coma Scale [GCS] score of $5)$ on Day 6 of her admission. About 5 h later, she experienced another episode of generalized tonic-clonic (GTC) seizure that lasted for 2-3 min and self-aborted.

Phenytoin level was checked and its corrected level was found to be sub-therapeutic at $5.1 \mathrm{mg} / \mathrm{L}$. The primary team obtained a neurology consult and a loading dose of IV phenytoin $1000 \mathrm{mg}$ was administered, followed by an increase of the maintenance dose of oral phenytoin from $200 \mathrm{mg} \mathrm{ON}$ to $300 \mathrm{mg}$ ON. A renal consult was also obtained, and the seizures were not thought to be related to the degree of azotemia at that point in time (serum urea $20.2 \mathrm{mmol} / \mathrm{L}$, serum sodium $134 \mathrm{mmol} / \mathrm{L}$, serum potassium $4.2 \mathrm{mmol} / \mathrm{L}$, serum creatinine $408 \mu \mathrm{mol} / \mathrm{L})$. Serum albumin was $25 \mathrm{~g} / \mathrm{L}$. A corrected phenytoin level of $23.7 \mathrm{mg} / \mathrm{L}$ was attained 1 day later (slightly above the upper limit of $20.0 \mathrm{mg} / \mathrm{L}$ ) and the patient had also regained a GCS score of 15 by then.

On the 13th day of treatment with ertapenem, the patient experienced another episode of seizure (1 min duration), presenting with upward rolling of eyes and left arm jerking. A repeat neurology consult was of the opinion that this was a breakthrough seizure due to sepsis. Although IV ertapenem was initially planned to be continued beyond 14 days, this was changed to IV meropenem after consulting with the resident pharmacist. This was subsequently changed to IV amikacin by the weekend on-call team in the evening due to concerns about the possibility of carbapenem-related seizures. The patient continued to have five further seizures on Day 14. 
At this point in time, two sequential repeat sets of blood cultures (aerobic and anaerobic) and urine cultures were both reported to be sterile, bar one bottle of blood culture. IV vancomycin was added while awaiting further identification. This eventually revealed itself as coagulase-negative staphylococcus as a result of skin contamination. Corrected serum calcium and phosphate were both rechecked and remained normal from admission.

The patient experienced another five episodes of seizures on the following day, Day 15. During this time, IV lorazepam $4 \mathrm{mg}$ bolus was given twice in addition to a loading regimen of IV valproate sodium (1200 mg over 2 days followed by $400 \mathrm{mg}$ IV TDS) after additional neurology input to control the status epilepticus.

Repeat serum phenytoin was found to be sub-therapeutic again at $7.25 \mathrm{mg} / \mathrm{L}$ (after correction for low albumin). Phenytoin was also converted from nasogastric route to IV $100 \mathrm{mg}$ TDS. The dose of phenytoin had been increased to $300 \mathrm{mg}$ ON via nasogastric route 10 days prior to this. Impaired absorption of phenytoin via the nasogastric route was thought to be the most likely cause for the sub-therapeutic levels $[5,6]$. The patient was not admitted to high dependency care at the family's request due to advanced age, advanced chronic kidney disease and expected poor outcome. GCS at this point was 6/15.

All antibacterials were stopped on Day 16 after review by the infectious disease consultant. The infectious disease team was of the opinion that ertapenem was the most likely underlying trigger for the seizures in view of the negative cultures and clinical improvement. The patient's blood pressure had not been compromised and her temperature had remained below $38{ }^{\circ} \mathrm{C}$ since admission 2 weeks previously. She had not had any episodes of hypoxia, tachyarrhythmia or hypoglycaemia under close observation in the acute admissions ward. There were no other concurrent medications which could potentially lower the seizure threshold.

Serum phenytoin remained sub-therapeutic at Day 16 at $8.46 \mathrm{mg} / \mathrm{L}$ (after correction), despite the conversion to IV route, and serum valproic acid levels were also sub-therapeutic at $7.05 \mathrm{mg} / \mathrm{L}$. Serum valproic acid levels remained low at Day 22 at $12.52 \mathrm{mg} / \mathrm{L}$ and serum phenytoin uncorrected was also low at $4.2 \mathrm{mg} / \mathrm{L}$. These low levels are likely due to the known drug-drug interactions between intravenous valproic acid and phenytoin. In spite of these sub-therapeutic levels, the patient remained seizure free from Day 16, 3 days after the ertapenem was stopped, and her GCS had improved dramatically to $13 / 15$ by Day 17 .

The IV anti-convulsant therapy was converted to nasogastric route after 10 days and the doses were subsequently weaned down and changed to oral levetiracetam monotherapy $250 \mathrm{mg}$ ON (dose adjusted for advanced renal failure and age) with no breakthrough seizures.
The patient was discharged 1 month later with slow stream rehabilitation and has returned home and remained seizure free to date on the same dose of levetiracetam.

Of note, although the dose of IV ertapenem was kept constant at $500 \mathrm{mg} \mathrm{q} 24 \mathrm{~h}$, we observed the patient had fluctuating renal function during her treatment with the antibacterial and the dips in $\mathrm{CrCl}$ were in keeping with the seizure intervals (Fig. 1). In addition, we noted that the seizures were preceded by episodes of delirium as defined by the Confusion Assessment Method (CAM) [7].

\section{Discussion}

Carbapenems are thought to induce seizures due to the similarity of the $\beta$-lactam ring to the structure of the inhibitory neurotransmitter $\gamma$-aminobutyric acid (GABA) and their antagonism of GABA receptors in the central nervous system [3]. However, seizures due to ertapenem are rare and are limited to few case reports to date $[3,8-$ $11]$.

Ertapenem is predominantly cleared by the kidneys $(\sim 80 \%)$ with half unchanged and half as a $\beta$-lactam openring metabolite, and the remaining $20 \%$ is excreted via the faeces $[12,13]$. The half-life of ertapenem is about $4.5 \mathrm{~h}$ in healthy individuals but it can be increased to $6.1-14.1 \mathrm{~h}$ in the presence of chronic renal impairment $[12,14]$. Some degree of renal dysfunction has been reported in a number of cases of neurotoxicity associated with ertapenem use [3, $11,14-16]$. It has been proposed that in the presence of uraemia, the blood-brain barrier is also more permeable and the protein binding of antibacterials is decreased [4, 11]. In addition, with the accumulation of endogenous uraemic toxins, a patient may then be more vulnerable to antibiotic-related neurotoxicity [4].

This is further compounded in elderly ( $>65$ years old) and very elderly ( $>80$ years old) patients who are known from population studies to have a high prevalence (about $25 \%$ for silent lacunes, and up to $95 \%$ for white matter changes/leucoaraiosis) of silent small vessel cerebrovascular disease [17]. This would directly increase the permeability of the blood-brain barrier to ertapenem and other carbapenems.

Miller and colleagues also suggested the presence of hypoalbuminaemia to be an independent risk factor for developing seizures with ertapenem compared with other carbapenems [3].

In the case that we have reported, the patient was a very elderly woman with underlying chronic kidney disease and low albumin levels $(20-30 \mathrm{~g} / \mathrm{L})$. As illustrated in Fig. 1, her renal function was further compromised due to acute kidney injury throughout her second treatment with ertapenem. We noted a trend with the occurrence of her 


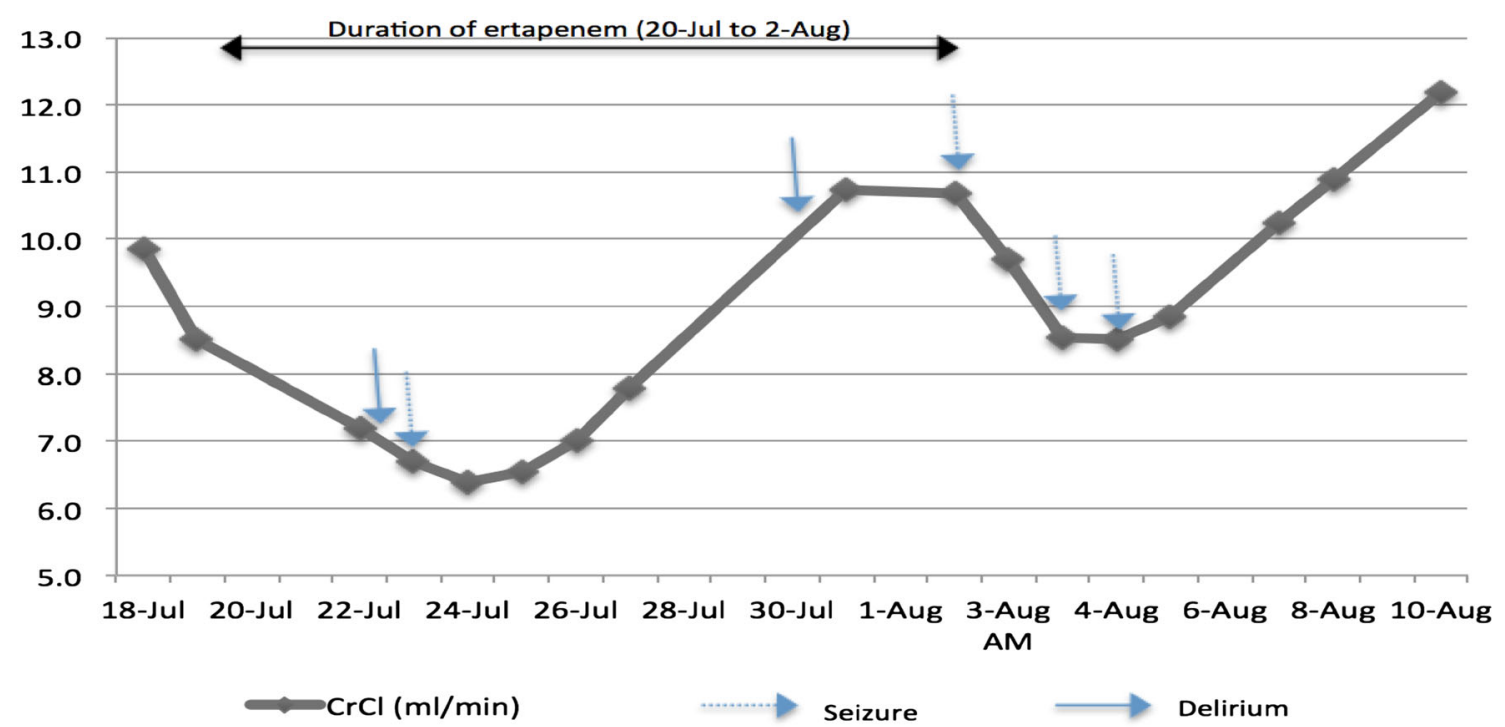

Fig. 1 Changes in creatinine clearance (Cockcroft-Gault equation) and occurrence of seizures

seizures corresponding with each drop in her $\mathrm{CrCl}$ level, despite being on a renal-adjusted dose of ertapenem. The onset of status epilepticus on Day 15 not only coincided with a drop in her estimated glomerular filtration rate (eGFR), but also with a drop in her serum phenytoin levels likely secondary to administration by the nasogastric tube. The nasogastric tube was inserted after the onset of delirium and first seizures on Day 6 to assist with nutrition and oral medications.

In a previous case report of a patient with stage 5 chronic kidney disease who developed neurotoxicity to ertapenem, the serum ertapenem level was detected to be much higher than the therapeutic minimum inhibitory concentration [16]. As such, we believe that the onset of delirium and seizure activity in relation to dips in our patient's $\mathrm{CrCl}$ could be a sign of spikes in serum ertapenem level.

In a study by Mistry and colleagues, the estimated clearance of ertapenem via the non-renal routes was lower in patients with kidney dysfunction, as compared to the estimated clearance of ertapenem via the non-renal route in healthy young adults with normal kidney function. This was based on the extrapolation of the roughly linear relationship between clearance of ertapenem and creatinine clerance to a creatinine clearance value of zero (i.e., the $y$-axis intercept of the graph, where the $y$-axis represents plasma clearance of total ertapenem and the $\mathrm{x}$-axis represents creatinine clearance). They proposed that this finding reinforces that part of non-renal clearance of ertapenem is facilitated by the kidneys and, in the presence of renal dysfunction, renal as well as non-renal clearance is affected adversely [12].

In addition, the presence of silent chronic microvascular ischaemic changes and small vessel disease on head CT performed in both admissions would further predispose our patient to neurotoxicity with ertapenem. Damage to the blood-brain barrier as a result of the ischaemic changes would likely lead to a higher concentration of ertapenem in brain tissue, the major determinant of CNS toxicity [2].

The number of days-to-onset of our patient's first seizure ( 8 days) with the first course of ertapenem was consistent with that reported in various case reports [3, 4, 9, 11]. With re-exposure to ertapenem, her seizures recurred despite being on an anti-epileptic drug. The last episode of seizure occurred 2 days after discontinuation of ertapenem and this could well be explained by taking into consideration the prolongation of ertapenem's half-life to 6.1-14.1 $\mathrm{h}$ in renal impairment and that about five half-lives are necessary for a drug to be completely cleared.

The patient remained seizure free thereafter despite repeated sub-therapeutic serum levels of phenytoin and valproic acid and conversion to single agent oral monotherapy over the following 4 weeks. Also, we noted a small rise in serum phosphate on 6 August 2013, 2 days after the episode of status epilepticus. As the patient remained well with no further seizures, this is likely to be secondary to the muscle damage as a result of the seizures and advanced chronic kidney disease, rather than the cause of the seizures.

In view of the above, the Naranjo Algorithm-a validated adverse drug reaction probability scale-scored 6 for our case study, suggesting that the seizure episodes were a probable adverse drug reaction caused by ertapenem [18].

\section{Naranjo Algorithm Score}

Our patient scored 6 based on the following answers to the questions in the algorithm. We have kept strictly to the points asked, and only answered yes or no when objective evidence were available to us: 
1. Yes. There have been previous conclusive reports.

2. Yes. The adverse event appeared after the suspected drug was administered.

3. Yes. The adverse reaction did improve when the drug was discontinued (even when the anti-epileptic drug serum levels were sub-therapeutic).

4. Yes. The reaction did re-appear when the drug was re-administered 2 days after the first discharge.

5. Yes. There are factors which could have caused the seizures on their own.

6. We don't know as no placebo was given to test this.

7. We don't know as no serum level of ertapenem was measured.

8. We don't know as there were no serial serum ertapenem levels available to answer this objectively.

9. Yes. The patient had the same reaction to the same drug in a previous admission.

10. We gave this a score of 0 as no EEG was done during or in between the seizure episodes.

\section{Conclusion}

There is a recognizable clinical pattern in the manifestation of ertapenem-induced delirium and seizures in an ill elderly patient with multiple morbidities. This is further signposted by dips in the $\mathrm{CrCl}$ if trended over time despite constant renal-adjusted administration of ertapenem.

We propose that this translates into spikes in serum levels of ertapenem as a result of further reduction in renal and extra renal clearance from complex drug-drug, drugdisease and drug-patient interactions. Discontinuation of ertapenem therapy should be considered if seizures develop [19], particularly if the organism is sensitive to other classes of antibacterials.

Although the incidence of ertapenem and other carbapenem-induced seizures are reported to be low in the general population, more work and studies are required to quantify the risks for specific sub-groups. A high index of suspicion is required to detect and report these unseen adverse drug reactions in the elderly and very elderly patients.

The findings of our retrospective case note audit for this case report have been submitted to the patient's Critical Medical Information Store (CMIS), part of Singapore's Pharmacovigilance Unit reporting system to avoid future exposure to ertapenem [20].

\section{Compliance with Ethical Standards}

Conflict of interest Huimin Lin and Samuel T.H. Chew declared that they have no conflicts of interest in the preparation of this case report.
Funding source No financial support was received for the conduct of this study, preparation of this manuscript or submission for publication.

Consent Written informed consent was obtained from the patient for publication of this case report. A copy of the written consent may be requested for review from the corresponding author.

Open Access This article is distributed under the terms of the Creative Commons Attribution-NonCommercial 4.0 International License (http://creativecommons.org/licenses/by-nc/4.0/), which permits any noncommercial use, distribution, and reproduction in any medium, provided you give appropriate credit to the original author(s) and the source, provide a link to the Creative Commons license, and indicate if changes were made.

\section{References}

1. Hammond ML. Ertapenem: a group 1 carbapenem with distinct antibacterial and pharmacological properties. J Antimicrob Chemother. 2004;53(Suppl 2):ii7-9.

2. Norrby SR. Carbapenems in serious infections: a risk-benefit assessment. Drug Saf. 2000;22(3):191-4.

3. Miller AD, Ball AM, Bookstaver PB, Dornblaser EK, Bennett CL. Epileptogenic potential of carbapenem agents: mechanism of action, seizure rates, and clinical considerations. Pharmacotherapy. 2011;31(4):408-23.

4. Chow KM, Hui AC, Szeto CC. Neurotoxicity induced by betalactam antibiotics: from bench to bedside. Eur J Clin Microbiol Infect Dis. 2005;24(10):649-53.

5. Phelps N. Management of phenytoin with enteral tube feeding. Mental Health Clin. 2012;2(5):108-9.

6. Decloedt E, Maartens G. Pitfalls of administering drugs via nasogastric tubes. S Afr Med J. 2009;99(3):148-9.

7. Inouye SK, van Dyck CH, Alessi C, Balkin S, Siegal AP, Horwitz RI. Clarifying confusion: the confusion assessment method: a new method for detection of delirium. Ann Inter Med. 1990;113(12):941-8.

8. Cannon JP, Lee TA, Clark NA, Setlak P, Grim SA. The risk of seizures among the carbapenems: a meta-analysis. J Antimicrob Chemother. 2014;69(8):2043-55.

9. Ong C, Chua AC, Tambyah PA, Yeoh SF. Seizures associated with ertapenem. Int J Antimicrob Agents. 2008;31(3):290.

10. Soštaric N, Beovic B, Maticic M. Ertapenem-associated seizures in a patient without prior CNS disorder or severe renal dysfunction. Int J Clin Pharmacol Ther. 2014;52(3):255-8.

11. Fica AE, Abusada NJ. Seizures associated with ertapenem use in patients with CNS disorders and renal insufficiency. Scand J Infect Dis. 2008;40(11-12):983-5.

12. Mistry GC, et al. Pharmacokinetics of ertapenem in patients with varying degrees of renal insufficiency and in patients on haemodialysis. J Clin Pharmacol. 2006;46(10):1128-38.

13. Livermore DM, Sefton AM, Scott GM. Properties and potential of ertapenem. J Antimicrob Chemother. 2003;52(3):331-44.

14. Shea YF, Mok MY, Cheng KC, Hon FK, Chu LW. Delayed recovery from ertapenem induced encephalopathy: case-report and a possible mechanism. Int J Clin Pharm. 2013;35(4):535-7.

15. Duquaine S, Kitchell E, Tate T, Tannen RC, Wickremasinghe IM. Central nervous system toxicity associated with ertapenem use. Ann Pharmacother. 2011;45(1):e6.

16. Wen MJ, Sung CC, Chau T, Lin SH. Acute prolonged neurotoxicity associated with recommended doses of ertapenem in 2 patients with advanced renal failure. Clin Nephrol. 2013;80(6):474-8. 
17. Roman GC, Erkinjuntti T, Wallin A, Pantoni L, Chui HC. Subcortical ischaemic vascular dementia. Lancet Neurol. 2002;1:426-36

18. Naranjo CA, et al. A method for estimating the probability of adverse drug reactions. Clin Pharmacol Ther. 1981;30(2):239-45.

19. Hawkey PM, Livermore DM. Carbapenem antibiotics for serious infections. BMJ. 2012;344:e3236.
20. Health Sciences Authority. Report Adverse Events related to Health Products. http://www.hsa.gov.sg/content/hsa/en/Health Products_Regulation/Safety_Information_and_Product_Recalls/ Report_Adverse_Events_related_to_health_products.html. Accessed 26 Apr 2015. 